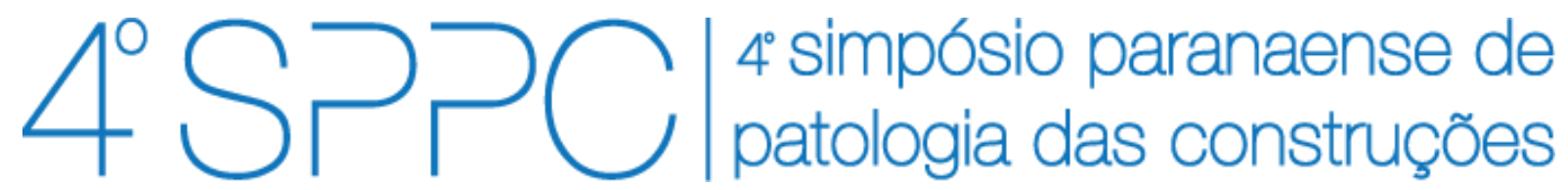

ISSN 2526-7248 artigo n. 4SPPC151, pp. 459-469, 2019

\title{
Influência do silicato de sódio neutro na degradação de pastas de cimento Portland submetidas ao ataque por ácido sulfúrico
}

Dobrovolski, Mateus Edilson Gomes"; Munhoz, Guilherme da Silva²; Nenevê, Bruno Leandro ${ }^{3}$; Dias, Roberto Luiz ${ }^{4}$; Godinho, Jayson Pereira ${ }^{5}$; Medeiros, Marcelo Henrique Farias ${ }^{6}$

${ }^{1}$ Eng. Civil, Mestrando em Engenharia de Construção Civil (PPGECC), Universidade Federal do Paraná (UFPR), mateusdobrovolski@gmail.com

${ }^{2}$ Eng. Civil, Mestrando em Engenharia de Construção Civil (PPGECC), Universidade Federal do Paraná (UFPR), munhoz.guilherme@outlook.com

${ }^{3}$ Eng. Civil, Mestrando em Engenharia de Construção Civil (PPGECC), Universidade Federal do Paraná (UFPR), brunoneneve@hotmail.com

${ }^{4}$ Eng. Civil, Mestrando em Engenharia de Construção Civil (PPGECC), Universidade Federal do Paraná (UFPR), robertoluizdias@gmail.com

${ }^{5}$ Eng. Ambiental e de Segurança do Trabalho, Doutorando em Engenharia de Construção Civil (PPGECC), Universidade Federal do Paraná (UFPR), jayson.godinho.eng@gmail.com

${ }^{6}$ Eng. Civil, Doutor em Engenharia Civil (USP), docente na Universidade Federal do Paraná (UFPR), marcelo.ufpr@gmail.com

Resumo: Visto que as instalações de tratamento de águas servidas estão sujeitas à corrosão induzida por micro-organismos devido ao ataque por ácido sulfúrico $\left(\mathrm{H}_{2} \mathrm{SO}_{4}\right)$, esse estudo buscou testar o silicato de sódio neutro $\left(\mathrm{Na}_{2} \mathrm{SiO}_{3}\right)$ como solução mitigadora do ataque ácido em pastas de cimento Portland. Foram moldados corpos de prova com diferentes teores de adição de $\mathrm{Na}_{2} \mathrm{SiO}_{3}(0 \%, 1 \%, 5 \%$ e $10 \%)$ e substituição de $35 \%$ do cimento Portland CP-V ARI por cinza volante classe C. Por fim, as amostras imersas em solução contendo $\mathrm{H}_{2} \mathrm{SO}_{4}$ foram comparadas com as amostras imersas em água saturada com cal. Nos resultados observou-se que a absorção de água e o índice de vazios dependeram do desenvolvimento da microestrutura e da velocidade da reação do cimento com o $\mathrm{H}_{2} \mathrm{SO}_{4}$, expresso pela recombinação química entre os íons sulfato e a portlandita. A degradação microestrutural foi comprovada com as análises de FT-IR.

Palavras-chave: Corrosão; Águas servidas; Ácido Sulfúrico; Silicato de sódio.

Abstract: Since that wastewater treatment plants are exposed to microorganisminduced corrosion due to sulfuric acid attack $\left(\mathrm{H}_{2} \mathrm{SO}_{4}\right)$, this study aimed to test neutral sodium silicate $\left(\mathrm{Na}_{2} \mathrm{SiO}_{3}\right)$ as a mitigating solution to the acid attack. Specimens were molded with different contents of $\mathrm{Na}_{2} \mathrm{SiO}_{3}(0 \%, 1 \%, 5 \%$ and $10 \%)$, and with $35 \%$ of CP-V ARI Portland cement replacement for class $\mathrm{C}$ fly ash. Finally, the samples immersed in sulfuric acid solution were compared with those immersed in saturated limewater. In the results, it was observed that the water absorption and the voids ratio depended on the development of the microstructure and the reaction kinetics between cement and $\mathrm{H}_{2} \mathrm{SO}_{4}$, expressed as the chemical recombination between sulphate and portlandite ions. The microstructural degradation was proven by the FT-IR analysis.

Keywords: Corrosion; Wastewater; Sulfuric acid; Sodium silicate. 
DOBROVOLSKI, M. E. G; MUNHOZ, G; NENEVÊ, B. L; DIAS, R. L; GODINHO, J. P; MEDEIROS, M. H. F. INFLUÊNCIA DO SILICATO DE SÓDIO NEUTRO NA DEGRADAÇÃO DE PASTAS DE CIMENTO PORTLAND SUBMETIDAS AO ATAQUE POR ÁCIDO SULFÚRICO. $4^{\circ}$ Simpósio Paranaense de Patologia das Construções (40 SPPC), artigo 4SPPC151, pp. 459 - 469, 2019.

\section{Introdução} DOI: $10.4322 / 2526-7248.068$

A corrosão induzida por micro-organismos proporciona o desgaste superficial de compostos cimentícios, caracterizado por descamação progressiva e desagregação, que ocorre tipicamente em ataque por ácido sulfúrico biogênico recorrente em sistemas de tratamento de esgoto [1-2]. Devido à biodegradação por bactérias anaeróbicas (disponíveis no fluxo de esgoto), os íons sulfato são convertidos em sulfeto de hidrogênio $\left(\mathrm{H}_{2} \mathrm{~S}\right)$, um gás tóxico, inflamável e agressivo ao concreto. Quando esse gás escoa para a atmosfera, as bactérias aeróbicas convertem-no em ácido sulfúrico $\left(\mathrm{H}_{2} \mathrm{SO}_{4}\right)$, aumentando ainda mais as chances de um processo corrosivo [3-5].

Após a recombinação química dos íons devido à biodegradação, o ácido sulfúrico reage com a Portlandita (hidróxido de cálcio - $\mathrm{Ca}(\mathrm{OH})_{2}$ ), e forma, em um primeiro momento, gipsita $\left(\mathrm{Ca}_{2} \mathrm{SO}_{4} \cdot 2 \mathrm{H}_{2} \mathrm{O}\right)$. Além disso, o ácido sulfúrico reage com 0 monossulfato de cálcio hidratado e o transforma em cristais de etringita, sendo este um produto secundário da reação [1-2][6]. Portanto, a reação do $\mathrm{H}_{2} \mathrm{SO}_{4}$ com os compostos hidratados do cimento forma produtos expansivos, que favorecem a fissuração e desagregação do elemento. Além disso, com o consumo do hidróxido de cálcio na reação, o $\mathrm{pH}$ do composto cimentício que era alcalino pode ser reduzido progressivamente. $\mathrm{O}$ ataque biogênico tem início quando o pH está abaixo de 9,0. Contudo, a redução do $\mathrm{pH}$ dos compostos cimentícios durante o ataque pode ser expressiva, podendo atingir valores inferiores a 1,0 [7-8].

Uma das alternativas para mitigar os efeitos do $\mathrm{H}_{2} \mathrm{SO}_{4}$ sobre os composto hidratados do cimento, são os componentes químicos cristalizantes [4][9]. As adições químicas cristalizantes, como o silicato de sódio $\left(\mathrm{Na}_{2} \mathrm{SiO}_{3}\right)$, têm sido uma opção que visa suprimir os efeitos da corrosão biogênica dos elementos de concreto causados pelo $\mathrm{H}_{2} \mathrm{SO}_{4}$. De modo geral, o princípio ativo cristalizante consiste na cicatrização das fissuras, tamponamento dos poros e redução da permeabilidade dos elementos, evitando a entrada de agentes agressivos [10]. Ao entrar em contato com os composto hidratados do cimento, o silicato de sódio sofre uma recombinação química, consumindo Portlandita e formando $\mathrm{C}-\mathrm{S}-\mathrm{H}$, reduzindo, dessa forma a permeabilidade da matriz cimentícia [10-12]. Assim, formulou-se uma hipótese de pesquisa questionando se as propriedades do $\mathrm{Na}_{2} \mathrm{SiO}_{3}$ permitiriam a redução da permeabilidade das amostras e evitariam a degradação por $\mathrm{H}_{2} \mathrm{SO}_{4}$.

Em suma, o objetivo do presente estudo consistiu em testar o silicato de sódio neutro em diferentes teores, buscando determinar qual o teor mais recomendado, e mitigar os efeitos da corrosão por $\mathrm{H}_{2} \mathrm{SO}_{4}$ em pastas de cimento Portland.

\section{Materiais e Métodos}

\subsection{Materiais}

As propriedades mitigadoras das pozolanas no combate à corrosão biogênica já foram constatadas na literatura [7][21]. Assim, para compreender o efeito do exato teor de cinza volante na mitigação do ataque por $\mathrm{H}_{2} \mathrm{SO}_{4}$, optou-se por simular um cimento CP IV e produzir um aglomerante contendo $65 \%$ de cimento Portland de alta resistência inicial (classificado como CP V-ARI pela norma NBR 16697 [13]) e 35\% de cinza volante classe C (NBR 12653 [14]). Observou-se que o cimento Portland CP V-ARI 
DOBROVOLSKI, M. E. G; MUNHOZ, G; NENEVÊ, B. L; DIAS, R. L; GODINHO, J. P; MEDEIROS, M. H. F. INFLUÊNCIA DO SILICATO DE SÓDIO NEUTRO NA DEGRADAÇÃO DE PASTAS DE CIMENTO PORTLAND SUBMETIDAS AO ATAQUE POR ÁCIDO SULFÚRICO. $4^{\circ}$ Simpósio Paranaense de Patologia das Construções (40 SPPC), artigo 4SPPC151, pp. 459 - 469, 2019. DOI: $10.4322 / 2526-7248.068$

apresenta massa específica de $3,09 \mathrm{~g} / \mathrm{cm}^{3}$ e superfície específica, determinada pelo método Blaine, de 0,445 $\mathrm{m}^{2} / \mathrm{g}$.

A cinza volante possui massa específica de 1,95 $\mathrm{g} / \mathrm{cm}^{3}$; superfície específica de $1.096 \mathrm{~m}^{2} / \mathrm{g}$, conforme método B.E.T; e atividade pozolânica de $408 \mathrm{mg} \mathrm{Ca}(\mathrm{OH}) 2 / \mathrm{g}$ pozolana, mensurada por meio do método Chapelle modificado. Na Tabela 1 é apresentada a composição química dos aglomerantes, enquanto que a Figura 1 ilustra o difratograma de raios- $x$ da cinza volante. A presença do halo amorfo reitera a reatividade da pozolana [15].

Tabela 1: Composição química dos aglomerantes.

\begin{tabular}{ccccccccccc}
\hline & \multicolumn{10}{c}{ Composição química (\%) } \\
\cline { 2 - 9 } Aglomerante & $\mathrm{CaO}$ & $\mathrm{SiO}_{2}$ & $\mathrm{Al}_{2} \mathrm{O}_{3}$ & $\mathrm{Fe}_{2} \mathrm{O}_{3}$ & $\mathrm{MgO}$ & $\mathrm{SO}_{3}$ & $\mathrm{~K}_{2} \mathrm{O}$ & $\begin{array}{c}\text { Demais } \\
\text { Óxidos }\end{array}$ & $\begin{array}{c}\text { Perda } \\
\text { ao } \\
\text { Fogo }\end{array}$ \\
\hline $\begin{array}{c}\text { Cimento CPV-ARI } \\
\begin{array}{c}\text { Cinza Volante } \\
\text { (Classe F) }\end{array}\end{array}$ & 60,61 & 18,71 & 4,26 & 2,95 & 3,52 & 3,12 & - & - & 3,34 \\
\hline
\end{tabular}

Nota ${ }^{1}-\mathrm{TiO}_{2}, \mathrm{Rb}_{2} \mathrm{O}, \mathrm{MnO}, \mathrm{ZnO}, \mathrm{CuO}, \mathrm{P}_{2} \mathrm{O}_{5}, \mathrm{Tm}_{2} \mathrm{O}_{3}$ e SrO.

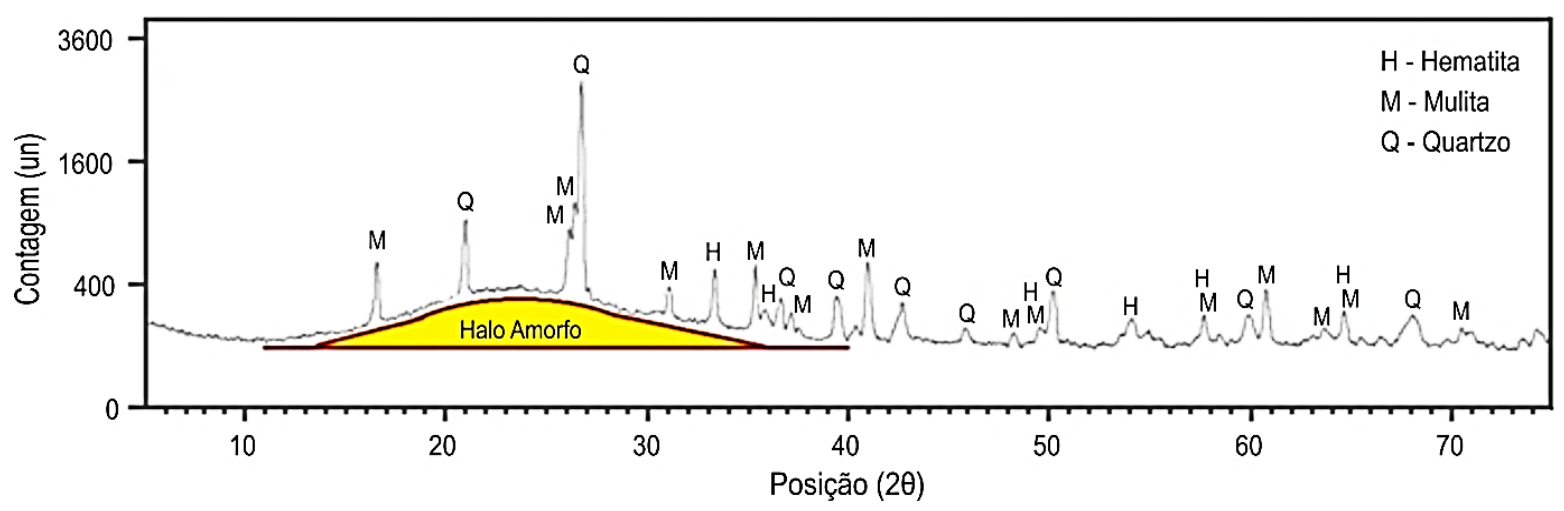

Figura 1: Difratograma de raios-x da cinza volante.

O aditivo superplastificante utilizado foi o MC-PowerFlow 1180, com densidade de $1,09 \mathrm{~g} / \mathrm{cm}^{3}$. Além disso, utilizou-se silicato de sódio neutro do tipo Q79, cuja especificação técnica é apresentada na Tabela 2.

Tabela 2: Especificação técnica de silicato de sódio neutro do tipo Q79.

\begin{tabular}{|c|c|c|c|c|c|c|}
\hline \multicolumn{7}{|c|}{ Especificação Técnica } \\
\hline $\begin{array}{c}\text { Densidade } \\
\left(\mathrm{g} / \mathrm{cm}^{3}\right)\end{array}$ & $\begin{array}{c}\text { Viscosidade } \\
\text { (cP) }\end{array}$ & $\begin{array}{l}\text { Óxido de } \\
\text { sódio } \\
(\%)\end{array}$ & $\begin{array}{l}\text { Oxido de } \\
\text { silício } \\
(\%)\end{array}$ & $\begin{array}{c}\text { Sólidos } \\
\text { Totais } \\
(\%)\end{array}$ & $\begin{array}{c}\text { Relação } \\
\mathrm{SiO}_{2} / \mathrm{Na}_{2} \mathrm{O} \\
---\end{array}$ & $\begin{array}{l}\text { Água } \\
(\%)\end{array}$ \\
\hline $1.39-1.42$ & $250-500$ & $8.5-9.2$ & $\begin{array}{c}27.8- \\
30.5\end{array}$ & $37.0-39.0$ & $3.27-3.31$ & $61.0-63.0$ \\
\hline
\end{tabular}


DOBROVOLSKI, M. E. G; MUNHOZ, G; NENEVÊ, B. L; DIAS, R. L; GODINHO, J. P; MEDEIROS, M. H. F. INFLUÊNCIA DO SILICATO DE SÓDIO NEUTRO NA DEGRADAÇÃO DE PASTAS DE CIMENTO PORTLAND SUBMETIDAS AO ATAQUE POR ÁCIDO SULFÚRICO. $4^{\circ}$ Simpósio Paranaense de Patologia das Construções (40 SPPC), artigo 4SPPC151, pp. 459 - 469, 2019.

\subsection{Métodos} DOI: $10.4322 / 2526-7248.068$

Todo o estudo foi desenvolvido em pasta de cimento, focando em investigar as modificações causadas pelo ataque por ácido sulfúrico. Para tanto, foram moldados corpos de prova cilíndricos de $22 \mathrm{~mm}$ de diâmetro por $44 \mathrm{~mm}$ de altura, conforme a norma NBR NM 43 [16]. A pasta estudada é resultante de um concreto de traço 1:1,90:2,47, com uma relação água/aglomerante de 0,45 - equivalente ao utilizado na construção de uma Estação de Tratamento de Esgoto (ETE) no estado do Paraná. Além do mais, na dosagem das pastas foram utilizados 4 teores de silicato de sódio neutro $\left(\mathrm{Na}_{2} \mathrm{SiO}_{3}\right): 0 \%$ (referência), $1 \%, 5 \%$ e $10 \%$. Todos os volumes de adição foram suplementares, e determinados em relação à massa de aglomerante. Tais teores foram escolhidos em função de uma lacuna na literatura, cujos valores testados são menores que $3 \%$ ou maiores que $20 \%$, em relação a massa de cimento [17-19]. Na Tabela 3 é apresentado o consumo de materiais para cada traço.

Tabela 3: Consumo de materiais $\left(\mathrm{kg} / \mathrm{m}^{3}\right)$ para cada traço

\begin{tabular}{cccccc}
\hline $\begin{array}{c}\text { Teor de } \\
\text { silicato }\end{array}$ & Cimento & Cinza volante & Água & Aditivo & Silicato \\
\hline $0 \%$ & 770,89 & 415,10 & 533,70 & 4,74 & 0,00 \\
$1 \%$ & 764,82 & 411,82 & 522,19 & 4,12 & 11,77 \\
$5 \%$ & 736,88 & 396,78 & 475,00 & 9,07 & 56,68 \\
$10 \%$ & 691,35 & 372,27 & 412,69 & 37,23 & 106,36 \\
\hline
\end{tabular}

Os corpos de prova foram moldados com 3 repetições para cada teor de silicato de sódio neutro $(0 \%, 1 \%, 5 \%$ e $10 \%)$, em ambos os ambientes de exposição (água saturada com cal e solução de ácido sulfúrico) e para três idades ( $0,14,56$ dias). $\mathrm{Na}$ Tabela 4 é apresentada a descrição dos tratamentos utilizados. Os resultados médios das propriedades das amostras analisadas passaram por análise estatística de variância, sendo as médias comparadas estatisticamente pelo teste de Tukey a $5 \%$ de significância, com o auxílio do software Sisvar.

Tabela 4: Definição dos tratamentos

\begin{tabular}{ccccccccc}
\hline Tratamento & $\begin{array}{c}\text { AS- } \\
\mathbf{0 0}\end{array}$ & $\begin{array}{c}\text { AS- } \\
\mathbf{0 1}\end{array}$ & $\begin{array}{c}\text { AS- } \\
\mathbf{0 5}\end{array}$ & $\begin{array}{c}\text { AS- } \\
\mathbf{1 0}\end{array}$ & $\begin{array}{c}\text { AG- } \\
\mathbf{0 0}\end{array}$ & $\begin{array}{c}\text { AG- } \\
\mathbf{0 1}\end{array}$ & $\begin{array}{c}\text { AG- } \\
\mathbf{0 5}\end{array}$ & $\begin{array}{c}\text { AG- } \\
\mathbf{1 0}\end{array}$ \\
\hline $\begin{array}{c}\text { Ambiente de } \\
\text { exposição }\end{array}$ & \multicolumn{3}{c}{ Solução de ácido sulfúrico } & & Água saturada com cal \\
$\begin{array}{c}\text { Teor de silicato de } \\
\text { sódio }\end{array}$ & $0 \%$ & $1 \%$ & $5 \%$ & $10 \%$ & $0 \%$ & $1 \%$ & $5 \%$ & $10 \%$ \\
\hline
\end{tabular}

Os espécimes foram desmoldados $24 \mathrm{~h}$ após a moldagem e submetidos a cura submersa em água saturada com cal por um período de 28 dias, a contar da data de moldagem. Em seguida, as amostras foram levadas para recipientes conforme seus respectivos ambientes de exposição, e imersas totalmente por um período de 56 dias. Os corpos de prova foram ensaiados logo após a desmoldagem (0 dias) e aos 14 e 56 dias de exposição. $\mathrm{O}$ pH da solução de ácido sulfúrico foi aferido quinzenalmente e mantido sempre em um intervalo entre 2,0 e 3,5, de modo a manter constante a agressividade do ataque por $\mathrm{H}_{2} \mathrm{SO}_{4}$ [20-22]. 
DOBROVOLSKI, M. E. G; MUNHOZ, G; NENEVÊ, B. L; DIAS, R. L; GODINHO, J. P; MEDEIROS, M. H. F. INFLUÊNCIA DO SILICATO DE SÓDIO NEUTRO NA DEGRADAÇÃO DE PASTAS DE CIMENTO PORTLAND SUBMETIDAS AO ATAQUE POR ÁCIDO SULFÚRICO. $4^{\circ}$ Simpósio Paranaense de Patologia das Construções (40 SPPC), artigo 4SPPC151, pp. 459 - 469, 2019.

\subsubsection{Absorção de água e índice de vazios} vazios

Para a determinação da absorção de água e do índice de vazios das pastas de cimento, seguiu-se o procedimento recomendado pela norma NBR 9778 [23].

\subsubsection{Espectroscopia de infravermelho por transformada de Fourier (FTIR)}

Para a análise de espectroscopia de infravermelho por transformada de Fourier (FTIR) foram coletadas amostras da superfície dos corpos de prova de concreto após 0, 14, 56 e 91 dias de exposição. A fim de paralisar as reações de hidratação do cimento, as amostras foram imersas em álcool etílico absoluto 99,5\% P.A por $24 \mathrm{~h}$ e levadas à estufa $40 \pm 5^{\circ} \mathrm{C}$ por $48 \mathrm{~h}$ [24-26]. Posteriormente, as amostras foram finamente moídas em um moinho de panelas, sendo analisado apenas o material passante na peneira de 325 mesh $(0,044 \mathrm{~mm})$. Para o ensaio foi utilizado um espectrofotômetro da marca Varian, modelo 640-IR com anexo de ATR (Attenuated total reflection reflectância total atenuada) da marca Pike, modelo Miracle com cristal de Zn-Se.

É importante destacar que a análise por FT-IR caracteriza a frequência de vibração das ligações químicas e, assim, consegue fazer inferências em relação aos compostos possivelmente presentes. Contudo, análises complementares são necessárias para confirmar a formação desses produtos, porém não fizeram parte desta fase da pesquisa experimental.

Além disso, o FT-IR é uma técnica semiquantitativa e, portanto, não possibilita a determinação da quantidade dos produtos formados. Desse modo, o ensaio foi interpretado comparando apenas as séries analisadas.

\section{Resultados e discussões}

\subsection{Absorção de água e índice de vazios}

Os resultados referentes à absorção de água das pastas de cimento ao longo do tempo estão ilustrados na Figura 2. Nas amostras expostas a água com cal (Figura 2A), a absorção de água reduziu ao longo do tempo, com exceção do tratamento AG-01, no qual não houve alteração estatisticamente significativa ao longo do tempo. Este comportamento está diretamente relacionado com a progressão da hidratação do cimento, das reações pozolânicas e da recombinação química do $\mathrm{Na}_{2} \mathrm{SiO}_{3}$ [10-11][2728]. Além do mais, quanto menor o teor de $\mathrm{Na}_{2} \mathrm{SiO}_{3}$, maior a reserva alcalina para ocorrência das reações pozolânicas [11][29]. Isto explica os resultados do tratamento AG-00: menor absorção de água após 56 dias.

Em contrapartida, a absorção de água das amostras expostas ao ácido sulfúrico aumentou ao longo do tempo (Figura 2B), sendo que amostras contendo $\mathrm{Na}_{2} \mathrm{SiO}_{3}$ obtiveram resultados piores se comparados à referência (AS-00), chegando a um aumento de $19 \%$ na absorção de água do tratamento contendo $1 \%$ de $\mathrm{Na}_{2} \mathrm{SiO}_{3}$ (AS01). Na Figura 3 estão ilustradas as amostras após 56 dias de exposição à solução de $\mathrm{H}_{2} \mathrm{SO}_{4}$ contendo diferentes teores de $\mathrm{Na}_{2} \mathrm{SiO}_{3}$, evidenciando a ação do agente agressivo. 
DOBROVOLSKI, M. E. G; MUNHOZ, G; NENEVÊ, B. L; DIAS, R. L; GODINHO, J. P; MEDEIROS, M. H. F. INFLUÊNCIA DO SILICATO DE SÓDIO NEUTRO NA DEGRADAÇÃO DE PASTAS DE CIMENTO PORTLAND SUBMETIDAS AO ATAQUE POR ÁCIDO SULFÚRICO. $4^{\circ}$ Simpósio Paranaense de Patologia das Construções (40 SPPC), artigo 4SPPC151, pp. 459 - 469, 2019. DOI: $10.4322 / 2526-7248.068$
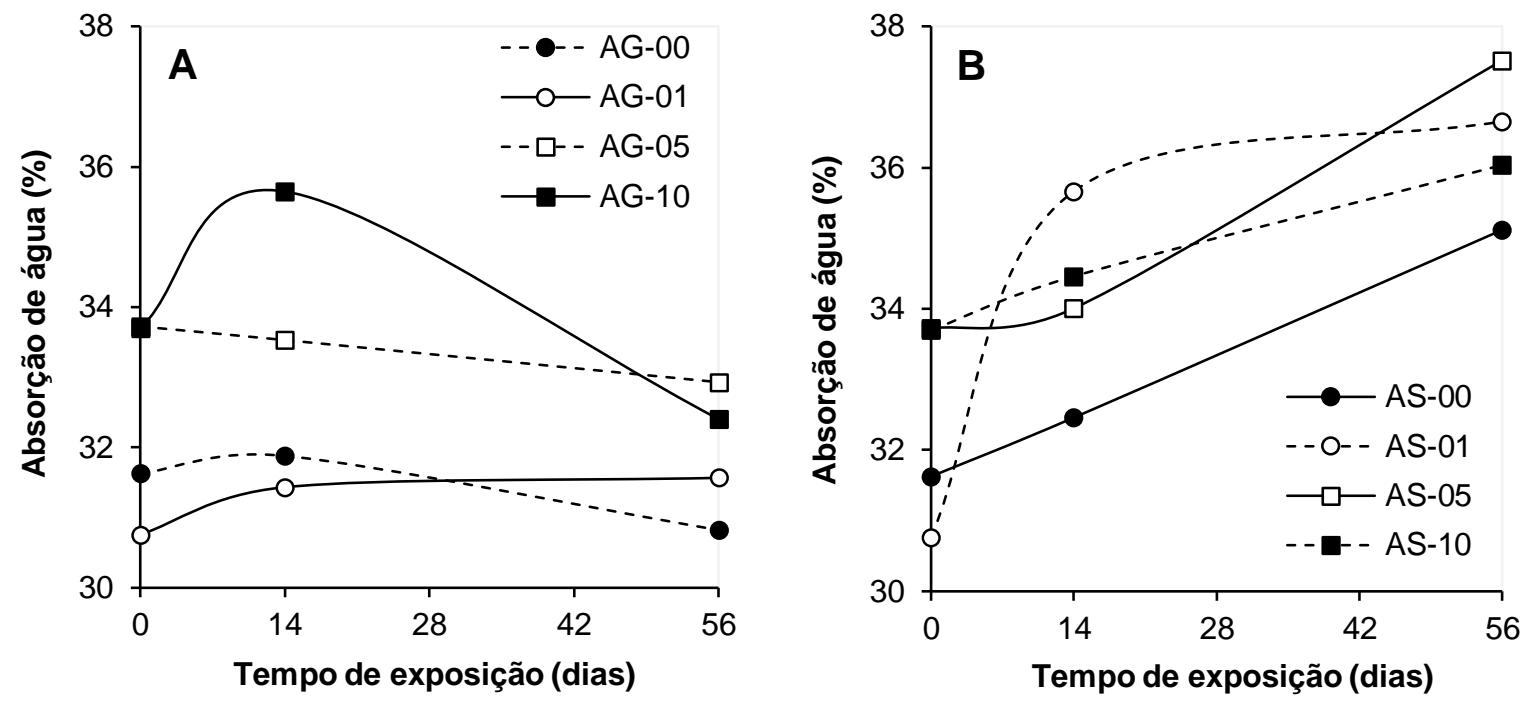

Figura 2: Absorção de água das pastas de cimento ao longo do tempo de (A) amostras expostas à água com cal e (B) amostras expostas a solução de $\mathrm{H}_{2} \mathrm{SO}_{4}$.
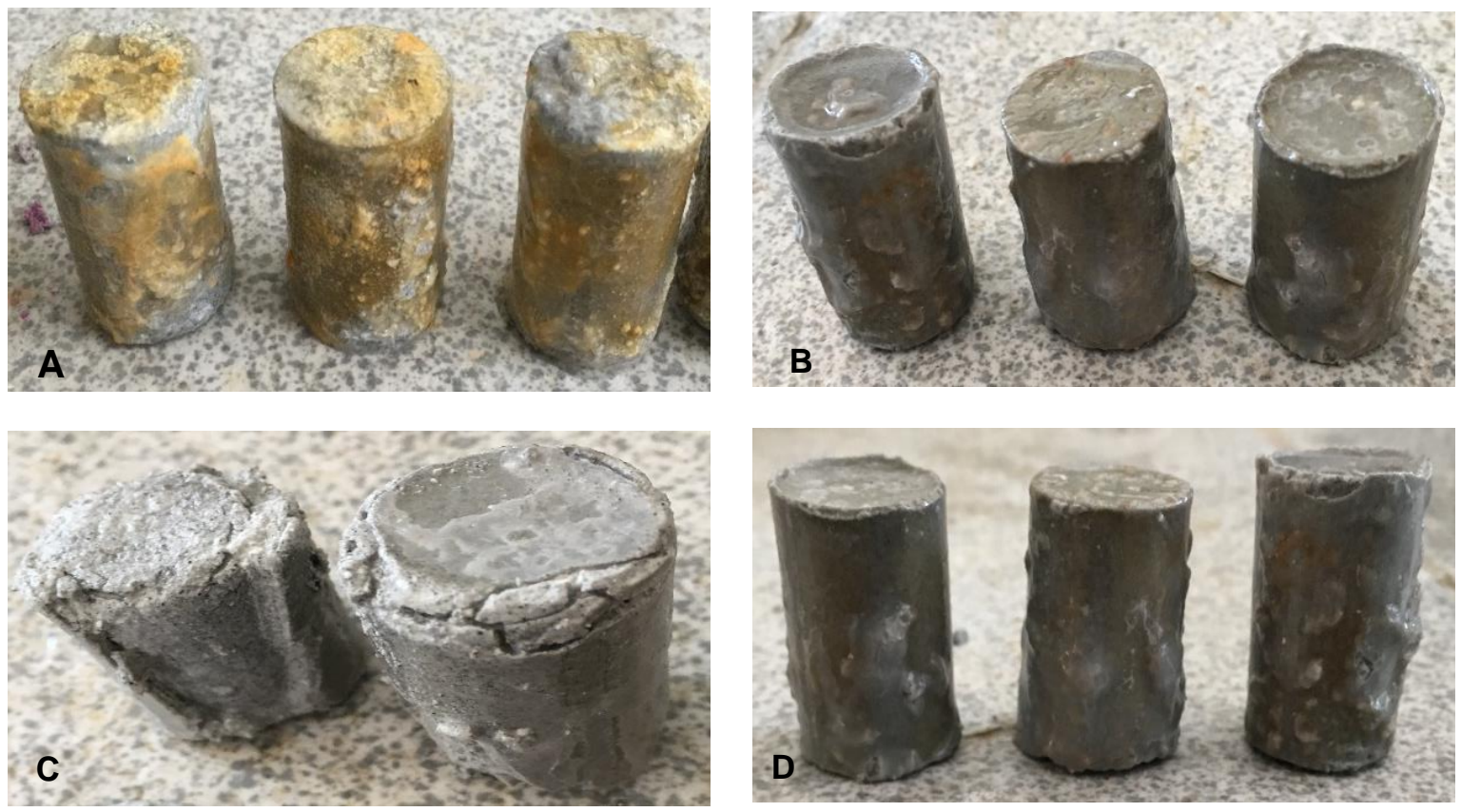

Figura 3: Amostras contendo (a) 0\%; (b) 1\%; (c) 5\% e (d) 10\% de $\mathrm{Na}_{2} \mathrm{SiO}_{3}$ após 56 dias de exposição à solução de $\mathrm{H}_{2} \mathrm{SO}_{4}$.

Na Figura 4 estão ilustrados os resultados de índice de vazios das pastas ao longo do tempo. Semelhante ao que ocorreu com a absorção de água, o índice de vazios das amostras expostas à água saturada com cal (Figura 4A) reduziu ao longo do tempo, em função da progressão da hidratação do cimento, da reação pozolânica e da recombinação química do $\mathrm{Na}_{2} \mathrm{SiO}_{3}$ [10-11][27-28]. 
DOBROVOLSKI, M. E. G; MUNHOZ, G; NENEVÊ, B. L; DIAS, R. L; GODINHO, J. P; MEDEIROS, M. H. F. INFLUÊNCIA DO SILICATO DE SÓDIO NEUTRO NA DEGRADAÇÃO DE PASTAS DE CIMENTO PORTLAND SUBMETIDAS AO ATAQUE POR ÁCIDO SULFÚRICO. $4^{\circ}$ Simpósio Paranaense de Patologia das Construções (40 SPPC), artigo 4SPPC151, pp. 459 - 469, 2019. DOI: $10.4322 / 2526-7248.068$

Do mesmo modo, o índice de vazios das amostras expostas ao $\mathrm{H}_{2} \mathrm{SO}_{4}$ (Figura 4B) aumentou ao longo do tempo. Entretanto, no tratamento AS-01 não houve alteração estatisticamente significativa do índice de vazios após 14 dias (semelhante ao tratamento AG-01), sendo este o tratamento com os melhores resultados. Isto ocorre devido a maior disponibilidade de $\mathrm{Ca}(\mathrm{OH})_{2}$, favorecendo a ocorrência de reações pozolânicas, as quais influenciam na integridade do concreto, e retardando a frente de ataque por $\mathrm{H}_{2} \mathrm{SO}_{4}$, a qual também consome a reserva alcalina [10-11][29].

\subsection{Espectroscopia de infravermelho por transformada de Fourier (FTIR)}

$\mathrm{Na}$ Figura 5 são apresentados os resultados da análise de Espectroscopia de Infravermelho por Transformada de Fourier (FTIR) aos 56 dias das pastas de cimento contendo $0 \%, 1 \%, 5 \%$ e $10 \%$ de $\mathrm{Na}_{2} \mathrm{SiO}_{3}$. É possível verificar que os valores de transmitância obtidados para a etringita e gipsita são maiores nas amostras expostas ao ácido sulfúrico, em especial nas pastas contendo $5 \%$ de $\mathrm{Na}_{2} \mathrm{SiO}_{3}$ (Figura $5 \mathrm{C}$ ) as quais apresentam os maiores picos correspondetes aos produtos da corrosão por $\mathrm{H}_{2} \mathrm{SO}_{4}$. Em compensação, as pastas contendo $1 \%$ de $\mathrm{Na}_{2} \mathrm{SiO}_{3}$ (Figura 5A), apresentaram os menores picos de transmitâncias referentes à etringita e gipsita. Ademais, a transmitância da Portlandita e do C-S-H é maior nas amostras expostas à água saturada com cal, evidenciando a ocorrência tanto da progressão da hidratação do cimento, como da reaçõe pozolânica e do ataque ácido [30-32].
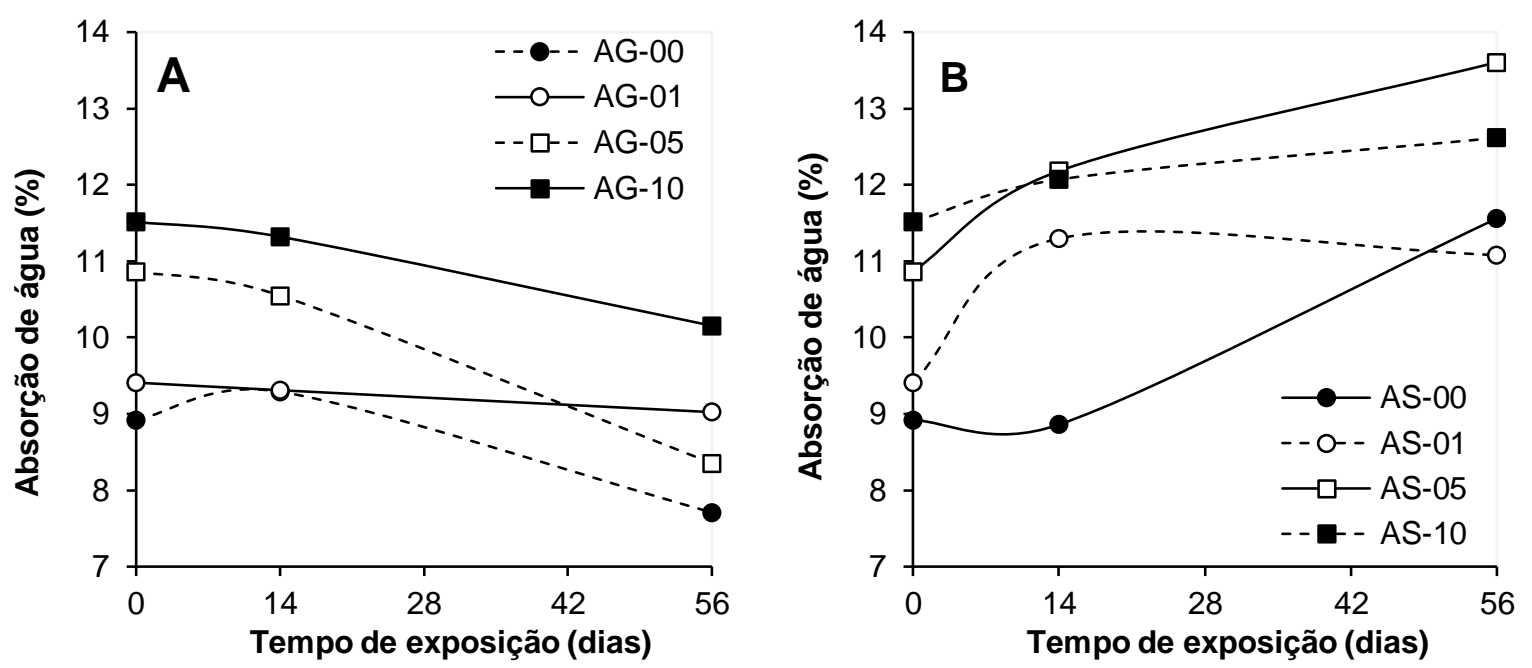

Figura 4: Índice de vazios das pastas de cimento ao longo do tempo de (A) amostras expostas à água com cal e (B) amostras expostas a solução de $\mathrm{H}_{2} \mathrm{SO}_{4}$. 
DOBROVOLSKI, M. E. G; MUNHOZ, G; NENEVÊ, B. L; DIAS, R. L; GODINHO, J. P; MEDEIROS, M. H. F. INFLUÊNCIA DO SILICATO DE SÓDIO NEUTRO NA DEGRADAÇÃO DE PASTAS DE CIMENTO PORTLAND SUBMETIDAS AO ATAQUE POR ÁCIDO SULFÚRICO. $4^{\circ}$ Simpósio Paranaense de Patologia das Construções (40 SPPC), artigo 4SPPC151, pp. 459 - 469, 2019. DOI: $10.4322 / 2526-7248.068$
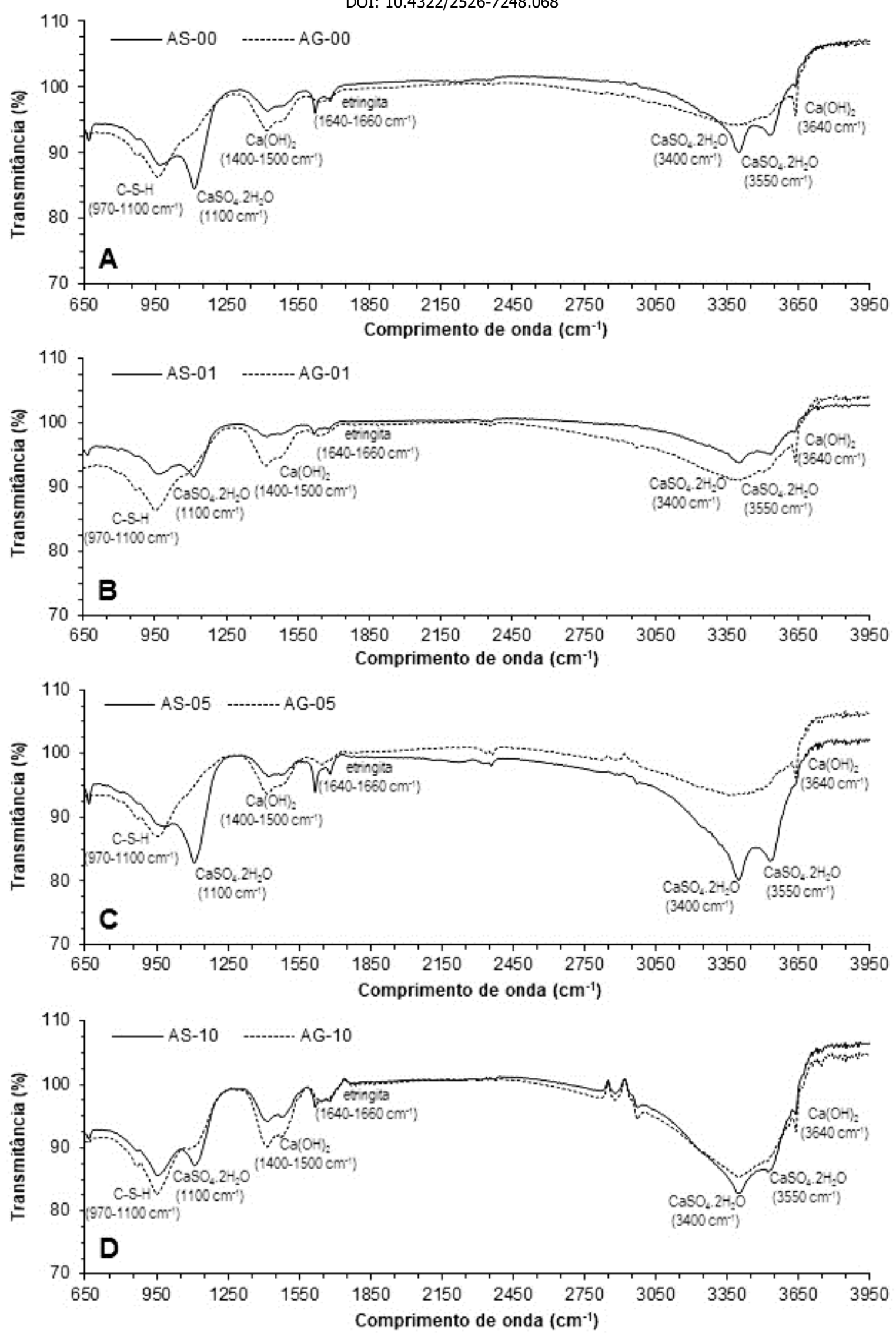

Figura 5: Análise de FT-IR das pastas de cimento contendo (A) $0 \%$, (B) $1 \%$, (C) $5 \%$ e (D) $10 \%$ de $\mathrm{Na}_{2} \mathrm{SiO}_{3}$ aos 56 dias. 
DOBROVOLSKI, M. E. G; MUNHOZ, G; NENEVÊ, B. L; DIAS, R. L; GODINHO, J. P; MEDEIROS, M. H. F. INFLUÊNCIA DO SILICATO DE SÓDIO NEUTRO NA DEGRADAÇÃO DE PASTAS DE CIMENTO PORTLAND SUBMETIDAS AO ATAQUE POR ÁCIDO SULFÚRICO. $4^{\circ}$ Simpósio Paranaense de Patologia das Construções (40 SPPC), artigo 4SPPC151, pp. 459 - 469, 2019.

\section{Conclusões} DOI: $10.4322 / 2526-7248.068$

Assim, com o objetivo de analisar a viabilidade do uso do $\mathrm{Na}_{2} \mathrm{SiO}_{3}$ neutro na mitigação do ataque por $\mathrm{H}_{2} \mathrm{SO}_{4}$ em ETEs, pôde-se concluir por meio dos ensaios realizados que:

- O teor de $1 \%$ de $\mathrm{Na}_{2} \mathrm{SiO}_{3}$ retardou a propagação do ataque por $\mathrm{H}_{2} \mathrm{SO}_{4}$ devido à maior disponibilidade de $\mathrm{Ca}(\mathrm{OH})_{2}$ aliado ao refinamento dos poros, sendo esse o teor mais eficiente quanto ao índice de vazios e FT-IR (menor pico de transmitância na banda referente à gipsita).

- A absorção de água e o índice de vazios das pastas dependeram do desenvolvimento da microestrutura e da velocidade da reação do concreto com o $\mathrm{H}_{2} \mathrm{SO}_{4}$, função da permeabilidade do concreto, concentração da solução e taxa de recombinação química entre os íons sulfato e a portlandita;

- O consumo da portlandita aconteceu sob três condições: reação pozolânica, devido a presença de cinza volante; recombinação química do $\mathrm{Na}_{2} \mathrm{SiO}_{3}$ (formando $\mathrm{C}-\mathrm{S}-\mathrm{H}$ ) e no ataque por $\mathrm{H}_{2} \mathrm{SO}_{4}$ (formando etringita secundária e gipsita);

Logo, com o desenvolvimento do presente estudo, recomenda-se a utilização de $1 \%$ de $\mathrm{Na}_{2} \mathrm{SiO}_{3}$ neutro para mitigar o ataque biogênico por $\mathrm{H}_{2} \mathrm{SO}_{4}$ que acomete, principalmente, as ETEs.

\section{Agradecimentos}

Os autores expressam a sua gratidão à Universidade Federal do Paraná (UFPR), especificamente ao Programa de Pós-Graduação em Engenharia de Construção Civil (PPGECC), ao Laboratório de Materiais e Estruturas (LAME), ao Centro de Microscopia Eletrônica (CME), ao grupo de pesquisa de Patologia e Recuperação das Construções (PRC), ao laboratório multiusuários de análises químicas (LAMAQ) da Universidade Tecnológica Federal do Paraná (UTFPR) e, também, às empresas Cia. de Cimentos Itambé, Buschle \& Lepper S.A, MC-Bauchemie Brasil e a Lubeco Ind. e Com. de Lubrificantes Vegetais Ltda, pela doação de material para a pesquisa.

\section{Referências}

[1] Hoppe Filho, J.; Rheinheimer, B.; Khoe, S. S.; Artigas, L. V.; Sabbag, A. F.; Medeiros, M. H. F. (2014) Degradação do concreto de uma Estação de Tratamento de Esgoto (ETE) por H2SO4 biogênico. Revista ALCONPAT. 4(2):87-99

[2] Jiang, G.; Wightman, E.; Donose, B. C.; Yuan, Z.; Bond, P. L.; Keller, J. (2014) The role of iron in sulfide induced corrosion of sewer concrete. Water Research, 49:166-174.

[3] Romanova, A.; Mahmoodian, M.; Alani, M. A. (2014) Influence and Interaction of Temperature, $\mathrm{H}_{2} \mathrm{~S}$ and $\mathrm{pH}$ on Concrete Sewer Pipe Corrosion. International Journal of Civil, Architectural. 8:592-595. 
DOBROVOLSKI, M. E. G; MUNHOZ, G; NENEVÊ, B. L; DIAS, R. L; GODINHO, J. P; MEDEIROS, M. H. F. INFLUÊNCIA DO SILICATO DE SÓDIO NEUTRO NA DEGRADAÇÃO DE PASTAS DE CIMENTO PORTLAND SUBMETIDAS AO ATAQUE POR ÁCIDO SULFÚRICO. $4^{\circ}$ Simpósio Paranaense de Patologia das Construções (40 SPPC), artigo 4SPPC151, pp. 459 - 469, 2019. DOI: $10.4322 / 2526-7248.068$

[4] Alani, A. M.; Faramarzi, A. (2014) An evolutionary approach to modelling concrete degradation due to sulphuric acid attack. Applied Soft Computing. 24:985-993.

[5] Yousefi, A.; Allahverdi, A.; Hejazi, P. (2014) Accelerated biodegradation of cured cement paste by Thiobacillus species under simulation condition. International Biodeterioration \& Biodegradation. 86:317-326.

[6] Yuan, H.; Dangla, P.; Chatellier, P.; Chaussadent, T. (2015) Degradation modeling of concrete submitted to biogenic acid attack. Cement and Concrete Research. 70:29-38.

[7] Mehta, P. K; Monteiro, P. J. M. (2006) Concrete - Microstructure, Properties and Materials. 3. ed. New York: McGraw Hill.

[8] Wei, S.; Jiang, Z.; Liu, H.; Zhou, D.; Sanchez-Silva, M. (2013) Microbiologically induced deterioration of concrete - A Review. Brazilian Journal of Microbiology. 44:1001-1007.

[9] Peyvandi, A.; Soroushian, P.; Balachandra, A. M.; Sobolev, K. (2013) Enhancement of the durability characteristics of concrete nanocomposite pipes with modified graphite nanoplatelets. Construction and Building Materials. 47:111-117.

[10] Medeiros, M. H. F.; Pereira, E.; Helene, P. (2012) Tratamento de superfície com silicato de sódio para concreto: penetração de cloretos e absorção de água. Revista ALCONPAT.2(3):155-168.

[11] Thompson, J. L.; Silsbee, M. R.; Gill, P. M.; Scheetz, B. E. (1997) Characterization of silicate sealers on concrete. Cement and Concrete Research. 27:1561-1567.

[12] Medeiros, M. H. F; (2008) Contribuição ao estudo da durabilidade de concretos com proteção superficial frente à ação de íons cloretos. Tese (Doutorado). Escola Politécnica da Universidade de São Paulo, São Paulo, Brasil.

[13] NBR 16697 (2018) Cimento Portland - Requisitos. Associação Brasileira De Normas Técnicas - ABNT. Rio de Janeiro, Brasil.

[14] NBR 12653 (2012) Materiais pozolânicos - Requisitos. Associação Brasileira De Normas Técnicas - ABNT. Rio de Janeiro, Brasil.

[15] Medeiros, M. H. F.; Raisdorfer, J. W.; Hoppe Filho, J.; Medeiros-Junior, R. A. (2017) Partial replacement and addition of fly ash in Portland cement: influences on carbonation and alkaline reserve. Journal of Building Pathology and Rehabilitation. 2:1-9.

[16] NBR NM 43 (2003) Cimento Portland - Determinação da pasta de consistência normal. Associação Brasileira De Normas Técnicas - ABNT. Rio de Janeiro, Brasil.

[17] Kazemian, S.; Huat, B. B. K.; Mohammed, T. A.; Barghchi, M. (2011) The effect of sodium silicate on cement-sodium silicate system grout. Modern Methods and Advances in Structural Engineering and Construction. 1:659-663.

[18] Raisdorfer, J. W.; Paim, A. S.; Santos, I. M. R.; Pereira, E.; Medeiros, M. H. F. (2013) Caracterização de concretos tratados com produtos cristalizantes para impermeabilização de piscinas com tratamento de água a base de Cloreto de sódio. Revista de Engenharia e Tecnologia. 5:111-123. 
DOBROVOLSKI, M. E. G; MUNHOZ, G; NENEVÊ, B. L; DIAS, R. L; GODINHO, J. P; MEDEIROS, M. H. F. INFLUÊNCIA DO SILICATO DE SÓDIO NEUTRO NA DEGRADAÇÃO DE PASTAS DE CIMENTO PORTLAND SUBMETIDAS AO ATAQUE POR ÁCIDO SULFÚRICO. $4^{\circ}$ Simpósio Paranaense de Patologia das Construções (40 SPPC), artigo 4SPPC151, pp. 459 - 469, 2019. DOI: $10.4322 / 2526-7248.068$

[19] Lone, I. H.; Muneeb, A.; Ahmad, J.; Jasim, S. M.; Ali, M.; Khan, M. A.; Malik, A. $\mathrm{H}$. (2015) Experimental study of effect of Sodium silicate $\left(\mathrm{Na}_{2} \mathrm{SiO}_{3}\right)$ on properties of concrete. International Journal of Civil Engineering and Technology. 6:39-47.

[20] Attiogbe, E. K.; Rizkalla, S. H. (1988) Response of Concrete to Sulfuric Acid Attack. ACI Materials Journal. 85(6):481-488.

[21] Monteny, J.; De Belie, N.; Taerwe, L. (2003) Resistance of different types of concrete mixtures to sulfuric acid. Materials and Structures. 36:242-249.

[22] Estokova, A.; Harbul'áková, V. O.; Luptàková, A.; Stevulová, N. (2012) Study of the deterioration of concrete influenced by biogenic sulphate attack. Procedia Engineering. 42:1731-1738.

[23] NBR 9778 (2005) Argamassa e concreto endurecido - Determinação da absorção de água, índice de vazios e massa específica. Associação Brasileira De Normas Técnicas - ABNT. Rio de Janeiro, Brasil.

[24] Pan, Z.; Li, D.; Yu, J.; Yang, N. (2002) Hydration products of alkali activated slagred mud cementitious material. Cement and Concrete Research. 32:357-362.

[25] Duart, M. A. (2008) Estudo da microestrutura do concreto adicionado de cinza da casca de arroz residual sem beneficiamento. Dissertação (Mestrado). Universidade Federal de Santa Maria. Santa Maria. Brasil.

[26] Capraro, A. P. B.; Braga, V.; Medeiros, M. H. F.; Hoppe Filho, J.; Bragança, M. O. G. P.; Portella, K. F.; Oliveira, I. C. (2017) Internal attack by sulphates in cement pastes and mortars dosed with different levels of pyrite. Journal of Building Pathology and Rehabilitation. 2(7):1-12.

[27] Ferreira, P. R. R.; Lima, M. S.; Meira, G. R. (2013) Concrete behaviour study in environments with sulphuric acid and chlorides. In: IX International Congress on Pathology and Repair of Structures, CINPAR. João Pessoa. Brasil.

[28] Song, Z.; Xue, X.; Li, Y.; Yang, J.; He, Z.; Shen, S.; Jiang, L.; Zhang, W.; Xu, L.; Zhang, H.; Qu, J.; Ji, W.; Zhang, T.; Huo, L.; Wang, B.; Lin, X.; Zhang, N. (2016) Experimental exploration of the waterproofing mechanism of inorganic sodium silicate-based concrete sealers. Construction and Building Materials. 104:276283.

[29] Baltazar, L.; Santana, J.; Lopes, B.; Rodrigues, M. P.; Correia, J. R. (2014) Surface skin protection of concrete with silicate-based impregnations: Influence of the substrate roughness and moisture. Construction and Building Materials. 70:191-200.

[30] Panzera, T. H; Sabariz, A. L. R; Borges, P. H. R; Vasconcelos, D. C. L; Wasconcelos, W. L (2010) Propriedades mecânicas de materiais compósitos à base de cimento Portland e resina epóxi. Cerâmica. 56:77-82.

[31] Lafuente B; Downs R. T; Yang H; Stone N (2015) The power of databases: the RRUFF project. In: Armbruster, T; Danisi, R. M. Highlights in Mineralogical Crystallography, Berlin, Germany, W. De Gruyter, 1-30.

[32] Piqué, T. M; Vázquez, A. (2012) Uso de Espectroscopía Infrarroja con Transformada de Fourier (FTIR) en el Estudio de la Hidratación del Cemento; Concreto Y Cemento. Investigación Y Desarrollo. 3:62-71. 